IPMU 09-0042

ICRR-Report-540

\title{
Constraining Light Gravitino Mass from Cosmic Microwave Background
}

\author{
Kazuhide Ichikawa ${ }^{1}$, Masahiro Kawasaki ${ }^{2,3}$, Kazunori Nakayama², \\ Toyokazu Sekiguchi ${ }^{2}$ and Tomo Takahashi ${ }^{4}$ \\ ${ }^{1}$ Department of Micro Engineering, Kyoto University, Kyoto 606-8501, Japan \\ ${ }^{2}$ Institute for Cosmic Ray Research, University of Tokyo, Kashiwa 277-8582, Japan \\ ${ }^{3}$ Institute for the Physics and Mathematics of the Universe, University of Tokyo, \\ Kashiwa, Chiba, 277-8568, Japan \\ ${ }^{4}$ Department of Physics, Saga University, Saga 840-8502, Japan
}

\begin{abstract}
We investigate the possibilities of constraining the light gravitino mass $m_{3 / 2}$ from future cosmic microwave background (CMB) surveys. A model with light gravitino with the mass $m_{3 / 2}<\mathcal{O}(10) \mathrm{eV}$ is of great interest since it is free from the cosmological gravitino problem and, in addition, can be compatible with many baryogenesis/leptogenesis scenarios such as the thermal leptogenesis. We show that the lensing of CMB anisotropies can be a good probe for $m_{3 / 2}$ and obtain an expected constraint on $m_{3 / 2}$ from precise measurements of lensing potential in the future CMB surveys, such as the PolarBeaR and CMBpol experiments. If the gravitino mass is $m_{3 / 2}=1 \mathrm{eV}$, we will obtain the constraint for the gravitino mass as $m_{3 / 2} \leq 3.2 \mathrm{eV}$ (95\% C.L.) for the case with Planck+PolarBeaR combined and $m_{3 / 2}=1.04_{-0.26}^{+0.22} \mathrm{eV}(68 \%$ C.L.) for CMBpol. The issue of Bayesian model selection is also discussed.
\end{abstract}




\section{Introduction}

One of the most important prediction of local supersymmetry (SUSY), or supergravity, is the existence of gravitino, the spin-3/2 superpartner of the graviton. Although the range of the gravitino mass $m_{3 / 2}$ can vary from a fraction of $\mathrm{eV}$ up to of an order of $\mathrm{TeV}$, depending on the scales of SUSY breaking, a light gravitino with $m_{3 / 2}<\mathcal{O}(10) \mathrm{eV}$ is of great interest since it is free from the cosmological gravitino problem. Furthermore, for some baryogenesis scenario to work such as thermal leptgenesis, high cosmic temperature is required, which favors the range of light mass for the gravitino. Thus, in this respect, the light gravitino would be attractive.

The determination of the gravitino mass is one of the most important issues to understand how supersymmetry is broken. Some authors have discussed this issue, in particular, focusing on probing the mass of the light gravitino with LHC experiment [1]. Since gravitino with very light mass of $m_{3 / 2}<100 \mathrm{eV}$ can play a role of warm dark matter (WDM) in the universe, cosmology would be a powerful tool as well. Some authors have obtained a constraint on the light gravitino mass from Lyman- $\alpha$ forest data in combination with WMAP [2,3] and its bound is $m_{3 / 2}<16 \mathrm{eV}$ at $2 \sigma$ level [2] $\#$. Although Lyman- $\alpha$ forest can be very useful as a cosmological probe, it may suffer from some systematic uncertainties [5,6]. Notice that the above mentioned limit crucially depends on the usage of Lyman- $\alpha$ forest data, thus in this respect, another independent cosmological probe of the light gravitino would be of great importance. Since light gravitino with the mass of interest here acts as WDM, it affects cosmic density fluctuations through two major effects. One is the change of radiation-matter equality due to the fact that light gravitions behave as relativistic component at earlier times. The second one is the effect of free-streaming which erases density contrast on the scale under which it can free-stream. Since these two effects can make great influence on cosmic microwave background (CMB) anisotropy, CMB can be a powerful probe of its mass. But in fact, when the gravitino is very light as $m_{3 / 2}<\mathcal{O}(10) \mathrm{eV}$, which is the range of the mass of interest here, its energy density do not have a large fraction of the total one in general to affect the CMB. Thus the mass of light gravitino can be mainly probed through the latter effect, free-streaming. The ultralight gravitino is almost relativistic at the time of the recombination, its effects imprinted on temperature anisotropy is not significant enough. However, by looking at the gravitational lensing of CMB photons, we can well probe the change of the gravitational potential driven during the intermediate redshift after the recombination when light gravitino comes to act as a non-relativistic component. Thus the lensed CMB is a very useful cosmological tool for investigating the mass of light gravitind\#2, which we discuss in this paper. Since much

\#1 Recently the authors of [3] also conducted a similar analysis using Lyman- $\alpha$ data combined with updated WMAP5 [4]. Although they did not report the constraint on the light gravitino mass, the SDSS Lyman- $\alpha$ dataset would be more effective in constraining $m_{3 / 2}$ than the dataset adopted in [2]. Thus an analysis optimized for the light gravitino model could give a severer constraint on $m_{3 / 2}$.

\#2 A similar argument has been made for massive neutrinos on the use of CMB lensing to constrain its mass Ref. [7-9]. 
more precise CMB experiments will be available in the near future, it is an interesting subject to investigate possible limits with such a future probe. Therefore, we in this paper study the possibilities of constraining its mass with future CMB observations without using other cosmological data. In particular, we focus on the lensing potential which can be reconstracted from $\mathrm{CMB}$ maps. As future $\mathrm{CMB}$ surveys, we consider Planck, PolarBeaR and CMBpol to discuss a possible constraint on the light gravitino mass.

The structure of this paper is as follows. We first briefly review a model which predicts light gravitino and its phenomenology in the early universe in Section 2 , In Section 3 we discuss the effects of the light gravitino on CMB anisotropy, paying particular attention to the lensing of CMB. We then present forecasts for constraints on the mass of light gravitino with future CMB surveys such as Planck, PolarBeaR and CMBpol in Section 4. In addition to the parameter estimation, we also discuss Bayesian model selection analysis for light gravitino model with future CMB surveys in Section 5. The final section is devoted to summary of this paper.

\section{Light gravitino: A model and its phenomenology in the early universe}

A light gravitino scenario is realized in the framework of gauge-mediated SUSY breaking (GMSB) models [10]. In GMSB models, the SUSY breaking effect in the hidden sector is transmitted to the minimal supersymmetric standard model (MSSM) sector through gauge-interactions, giving superparticles TeV scale masses. As an example, let us consider a model where the SUSY breaking field $S$ couples to $N$ pairs of messenger particles $\psi$ and $\bar{\psi}$, which transform as fundamental and anti-fundamental representations of $\mathrm{SU}(5)$, having a superpotential $W=\lambda S \psi \bar{\psi}$ with coupling constant $\lambda$ (in the following we set $\lambda=1$ for simplicity). The superfield $S$ has a vacuum expectation value as $\langle S\rangle=M+F_{S} \theta^{2}$. Here $F_{S}$ gives SUSY breaking scale, which is related to the garvitino mass $m_{3 / 2}$ through the relation $F_{S}=\sqrt{3} m_{3 / 2} M_{P}$, with $M_{P}$ being the reduced Planck energy scale, for vanishing cosmological constant. In this model, gaugino masses $M_{a}(a=1,2,3$ are gauge indices) and sfermion masses squared $m_{\tilde{f}_{i}}^{2}$ at the messenger scale are given by

$$
\begin{gathered}
M_{a}=N\left(\frac{\alpha_{a}}{4 \pi}\right) \Lambda_{\mathrm{mess}} \\
m_{\tilde{f}_{i}}^{2}=2 N \sum_{a}\left(\frac{\alpha_{a}}{4 \pi}\right)^{2} C_{a}^{(i)} \Lambda_{\mathrm{mess}}^{2}
\end{gathered}
$$

where $\alpha_{a}$ denotes the gauge coupling constants, $C_{a}^{(i)}$ are Casimir operators for the sfermion $\tilde{f}_{i}$ and the messenger scale is given by $\Lambda_{\text {mess }}=F_{S} / M$. In order to obtain TeV scale masses, $\Lambda_{\text {mess }} \sim 100 \mathrm{TeV}$ is required, but still the SUSY breaking scale $F_{S}$, or gravitino mass $m_{3 / 2}$ can take wide range of values as $1 \mathrm{eV} \lesssim m_{3 / 2} \lesssim 10 \mathrm{GeV}$. The upper bound comes from the requirement that the gravity-mediation effect does not dominate. On the other hand, there 
also exists a lower bound on the gravitino mass in order not to destabilize the messenger scalar and lead to the unwanted vacuum. This requires $m_{3 / 2} \gtrsim \mathcal{O}(1) \mathrm{eV}$.

However, if cosmological effects of the gravitino are taken into account, not all of its mass range is favored. This is because gravitinos are efficiently produced at the reheating era and it can easily exceed the present dark matter abundance unless the reheating temperature $T_{R}$ is very low [12]. This is problematic since many known leptogenesis/baryogenesis scenarios require high enough reheating temperature which may conflict with the upper bound coming from the gravitino problem. In particular, thermal leptogenesis scenario [13], which requires $T_{R} \gtrsim 10^{9} \mathrm{GeV}$, seems to be inconsistent with the gravitino problem except for the very light gravitino mass range $m_{3 / 2} \lesssim 100 \mathrm{eV}$. As we will see, gravitinos with such a small mass are thermalized in the early Universe. Thus their abundance does not depend on the reheating temperature and also it is smaller than the dark matter density for $m_{3 / 2} \lesssim 100 \mathrm{eV}$. This is the reason why we pay particular attention to a light gravitino scenario.

Having described that the light gravitino scenario is appealing from the view point of cosmological gravitino problem, next we briefly discuss thermal evolution of the light gravitino in the early universe. Gravitinos are relativistic well before the recombination. In such a case, the energy density of the gravitino is parameterized by the effective number of neutrino species, and it is given by

$$
N_{3 / 2}=\frac{\rho_{3 / 2}}{\rho_{\nu}}=\left(\frac{T_{3 / 2}}{T_{\nu}}\right)^{4}=\left(\frac{g_{* \nu}}{g_{* 3 / 2}}\right)^{4 / 3},
$$

where $\rho_{\nu}\left(\rho_{3 / 2}\right)$ and $g_{* \nu}\left(g_{* 3 / 2}\right)$ are, respectively, the energy density and the effective degrees of freedom of neutrinos (gravitinos) evaluated at the epoch when neutrinos (gravitinos) have decoupled from thermal plasma while they are still relativistic. In the standard cosmology, $g_{* \nu}=10.75$. Temperatures of neutrino and gravitino are represented by $T_{\nu}$ and $T_{3 / 2}$. From Eq. (3) we can calculate the temperature of gravitino at present:

$$
T_{3 / 2}=\left(N_{3 / 2}\right)^{1 / 4} T_{\nu}=1.95\left(N_{3 / 2}\right)^{1 / 4}[\mathrm{~K}],
$$

where we have adopted the temperature of neutrino in the standard cosmology at the second equality. Eventually the gravitino loses its energy and becomes non-relativistic due to the Hubble expansion. Its present energy density is given by

$$
\omega_{3 / 2} \equiv \Omega_{3 / 2} h^{2}=0.13\left(\frac{m_{3 / 2}}{100 \mathrm{eV}}\right)\left(\frac{90}{g_{* 3 / 2}}\right) .
$$

For later convenience, we also define the fraction of gravitino in the total dark matter density $\omega_{\mathrm{dm}}$ as

$$
f_{3 / 2} \equiv \frac{\omega_{3 / 2}}{\omega_{\mathrm{dm}}}
$$

In the following, we assume that dark matter consists two components: light gravitino, which acts as warm dark matter, and CDM. As CDM component, the Peccei-Quinn axion, 
a messenger baryon proposed in [11] and so on can be well-fitted into the framework of light gravitino.

Thus in order to evaluate the relic abundance of light gravitino, we must know the value of the effective degrees of freedom of relativistic particles at the freeeze-out epoch, $g_{* 3 / 2}$. Since the production and/or destruction of the light gravitino due to scattering processes are known to be inefficient for the low temperature regime, in which we are interested, the gravitino maintains equilibrium with thermal bath through the decay and inverse-decay processes [12,14], schematically represented by $a \leftrightarrow b+\tilde{G}$, where $b$ is the standard model (SM) particle and $a$ is its superpartner. As the temperature decreases, particles in thermal bath $(b$ and $\tilde{G}$ ) lose an ability to create a heavy particle $(a)$. Then gravitinos decouple from thermal plasma after the time when $a$ decays into $b$ and $\tilde{G}$ without inverse creation processes.

In order to see these processes in detail, we must solve the Boltzmann equation which governs time evolution of the system. The Boltzmann equation for the gravitino number density $n_{3 / 2}$ is given by

$$
\dot{n}_{3 / 2}+3 H n_{3 / 2}=\sum_{a, b} \Gamma(a \rightarrow b \tilde{G})\left\langle\frac{m_{a}}{E_{a}}\right\rangle n_{a}\left(1-\frac{n_{3 / 2}}{n_{3 / 2}^{(\mathrm{eq})}}\right),
$$

where $H$ is the Hubble expansion rate and $\left\langle m_{a} / E_{a}\right\rangle$ represents thermally averaged Lorentz factor with $m_{a}$ and $E_{a}$ being the mass and energy of the particle $a$, respectively. $\Gamma(a \rightarrow b \tilde{G})$ is the decay width of $a$ into $b$ and $\tilde{G}$ and superscript (eq) denotes its equilibrium value. As an example, the decay rate of the stau $(\tilde{\tau})$ into tau $(\tau)$ and gravitino is given by

$$
\Gamma(\tilde{\tau} \rightarrow \tau \tilde{G})=\frac{1}{48 \pi} \frac{m_{\tilde{\tau}}^{5}}{m_{3 / 2}^{2} M_{P}^{2}}
$$

and similar expressions hold for other particles. By solving this equation, one obtains the final gravitino abundance which can be represented in terms of the gravitino-to-entropy ratio, $Y_{3 / 2}=\left(n_{3 / 2} / s\right)(t \rightarrow \infty)$ and this translates into $g_{* 3 / 2}$ through the relation $Y_{3 / 2}=$ $0.417 / g_{* 3 / 2}$. In Fig. 1, the value of $g_{* 3 / 2}$ is shown as a function of $m_{3 / 2}$ for several values of $\Lambda_{\text {mess }}$. We have adopted $\Lambda_{\text {mess }}=50,100,200 \mathrm{TeV}$ and $N=1$ and ignored running of the masses from the messenger scale down to the weak scale for simplicity.

We can understand these results intuitively. As the gravitino mass increases, the decay width becomes smaller, and hence the equilibrium lasts for rather shorter duration. This leads to higher freeze-out temperature of the gravitino, which corresponds to large $g_{* 3 / 2}$. On the other hand, larger $\Lambda_{\text {mess }}$ leads to heavier sparticle masses, which obviously makes the time of freeze-out of the gravitino earlier, and hence higher $g_{* 3 / 2}$. However, as seen in the figure, the dependence on these parameters are not so strong and we can safely set $g_{* 3 / 2} \simeq 90$ even if we take into account model uncertainties. 


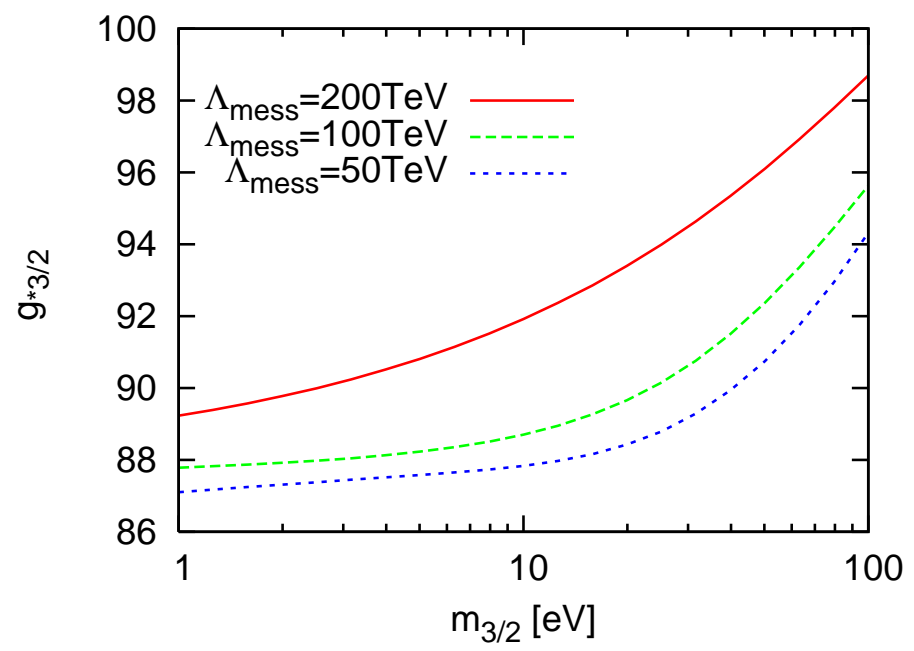

Figure 1: $g_{* 3 / 2}$ as a function of $m_{3 / 2}$ for several values of $\Lambda_{\text {mess }}$.

\section{Effects of light gravitino on CMB}

In this section we discuss how light gravitino affects the structure formation and the CMB anisotropies. Since light gravitino basically acts as WDM, it is characterized by two quantities, its mass and number density. The number density is determined by the effective number of degrees of freedom at the time of the decoupling, i.e. $g_{* 3 / 2}$. Since, as we have seen in the previous section, $g_{* 3 / 2}$ has only mild dependence on $m_{3 / 2}$, we can take $g_{* 3 / 2}=90$ as the representative value, which corresponds to

$$
N_{3 / 2}=0.059,
$$

from Eq. (3). We assume Eq. (9) as a fiducial value throughout this section, except for the last paragraph. We also assume that the universe is flat, dark energy is a cosmological constant and the primordial fluctuations are adiabatic and its power spectrum obeys a power-law without tensor perturbations. The fiducial values for cosmological parameters are adopted from the the recent result of WMAP5 [4], except that we consider mixed dark matter scenarios $\omega_{\mathrm{dm}}=\omega_{c}+\omega_{3 / 2}=0.1099$, instead of $\omega_{c}=0.1099$ where $\omega_{c}$ is the density parameter for cold dark matter $(\mathrm{CDM})$. By varying $m_{3 / 2}$ or $f_{3 / 2}$, we can see the effects of light gravitino on structure formation and CMB anisotropies. Moreover, we assume that neutrinos are massless in the most part of this paper. We will make some comments on the case where massive neutrinos are also included in Section 6 ,

As briefly discussed in the introduction, the effects of WDM on structure formation can be understood by considering following two main aspects: (i) the change of the energy contents of the universe, or the epoch of matter-radiation equality (unperturbed background evolutions), (ii) the erasure of perturbations on small scales via free-streaming 
(perturbation evolutions). The first point is due to the fact that WDM behaves as relativistic component at early times but non-relativistic one at late times. Thus it changes the time of matter-radiation equality depending on the mass. It alters the evolution of gravitational potential and drives the integrated Sachs-Wolfe (ISW) effect in the CMB temperature anisotropy. However, in the case of light gravitino in which we are interested, its abundance is so small $N_{3 / 2}=0.059$ that the epoch of radiation-matter equality is scarcely affected, even when we compare the two opposite limits, $f_{3 / 2}=0\left(m_{3 / 2}=0 \mathrm{eV}\right)$ and $f_{3 / 2}=1\left(m_{3 / 2}=86 \mathrm{eV}\right)$, with fixed $\omega_{\mathrm{dm}}=0.1099$. Therefore it is almost impossible to constrain the gravitino mass from unlensed CMB anisotropies, even when the ideal observations (cosmic variance limeted survey) are available.

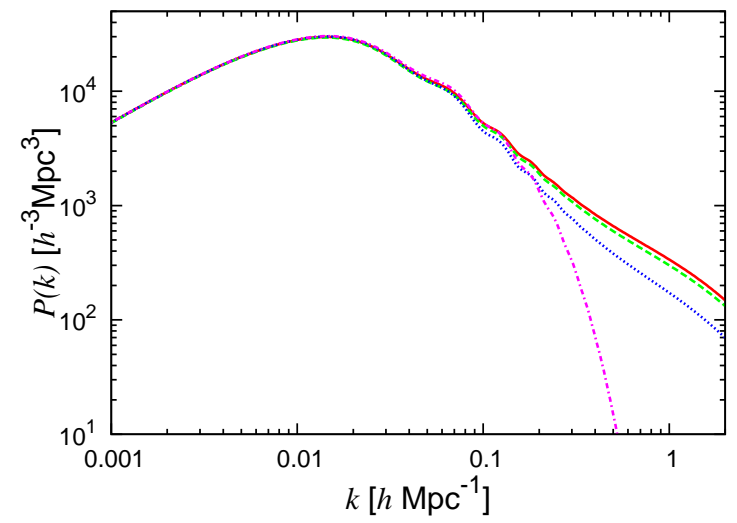

Figure 2: Matter power spectra $P(k)$ for several values of $m_{3 / 2}$. We have plotted the cases with $m_{3 / 2}=0 \mathrm{eV}$ (solid red line), $1 \mathrm{eV}$ (dashed green line), $10 \mathrm{eV}$ (dotted blue line), 86 $\mathrm{eV}$ (dot-dashed magenta line). In all cases we fixed the total dark matter density and energy density of gravitino as $\omega_{\mathrm{dm}}=0.1099$ and $N_{3 / 2}=0.059$, respectively. We adopt HALOFIT [30] in calculating nonlinear corrections.

Possible constraints on the gravitino mass almost come from the second point. Light gravitino free-streams to erase cosmic density fluctuations while it is relativistic. On scales smaller than the free streaming length of gravitino, fluctuations of matter and hence gravitational potential are erased. To see the effect, we show matter power spectra $P(k)$ for several values of $m_{3 / 2}$ with $\omega_{\mathrm{dm}}$ being fixed in Fig. 2. We take $m_{3 / 2}=0 \mathrm{eV}$ (solid red), $m_{3 / 2}=1 \mathrm{eV}$ (dashed green), $m_{3 / 2}=10 \mathrm{eV}$ (dotted blue) and $m_{3 / 2}=86 \mathrm{eV}$ (dot-dashed magenta). As seen from the figure, as $m_{3 / 2}$ increases, the suppression of $P(k)$ becomes larger on small scales, while on large scales the amplitude of $P(k)$ is unaffected regardless of the value of $m_{3 / 2}$. With more careful observation we can notice that when $m_{3 / 2}$ is small, the suppression of the power is small, but the scale under which $P(k)$ is suppressed becomes large. On the other hand, when $m_{3 / 2}$ is large, the suppression is also large, however the free-streaming scale becomes small. These can be simply understood as follows. When the mass of gravitino is small, gravitino can erase cosmic structure up to 
large scales. However, the smallness of the mass in turn indicates that gravitino is minor component in the contents of energy density and gravitationally irrelevant. Thus density fluctuations are less suppressed. For the case of larger mass, the opposite argument holds.
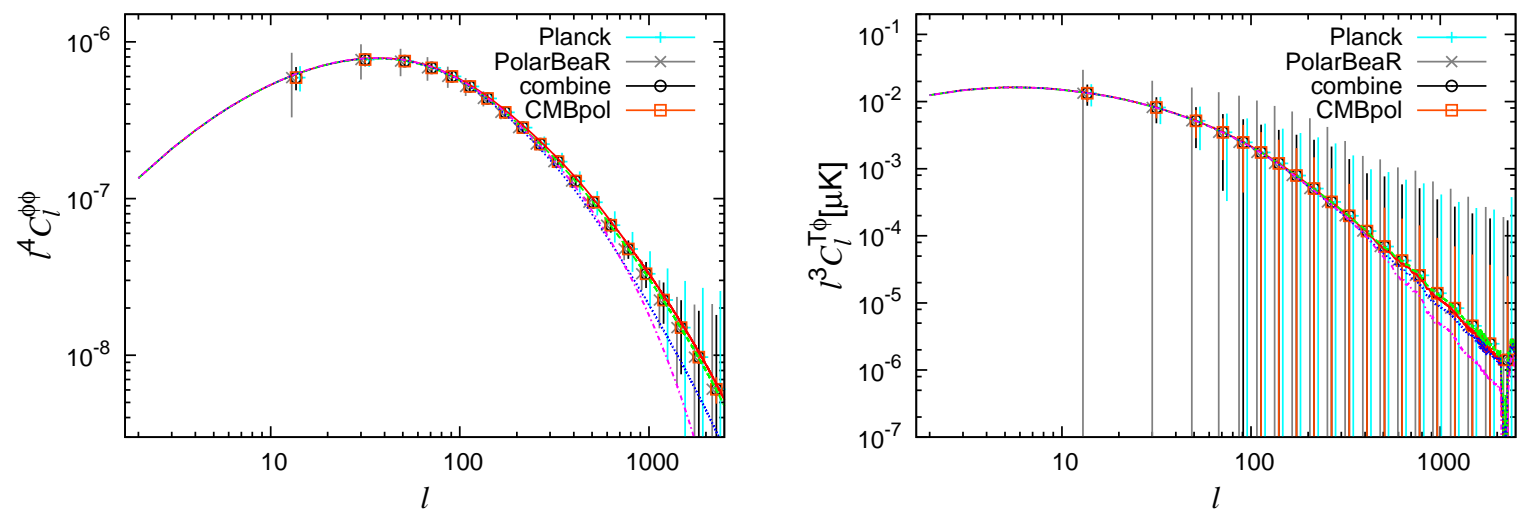

Figure 3: The angular power spectra of lensing potential $C_{\ell}^{\phi \phi}$ (Left) and its correlation with temperature $C_{\ell}^{T \phi}$ (Right). The values of $m_{3 / 2}$ are same as in Figure 2, The sensitivities of future CMB surveys used in our analysis, Planck, PolarBeaR and CMBpol, are also shown as points with error bars. Notice that the bins are linearly and logarithmically spaced in $\ell$ for $\ell \leq 100$ and $\ell>100$, respectively. For visual reason, data points for Planck and PolarBeaR are slightly displaced horizontally.

Now we move on to discuss how the suppression of matter fluctuations changes the lensed CMB anisotropy. CMB photons last-scattered at the decoupling epoch, while traveling to the present epoch, are deflected by the gravitational potential $\Phi(\mathbf{r}, \eta)$ generated by the matter fluctuations (For a recent review see e.g. [15]). The lensing potential $\phi$ is given by

$$
\phi(\hat{n})=-2 \int_{0}^{\chi_{*}} d \chi \frac{\chi_{*}-\chi}{\chi_{*} \chi} \Phi(\chi \hat{n}, \eta(\chi))
$$

where $\chi$ is the comoving distance along the line of sight, $\chi_{*}$ is the comoving distance to the last scattering surface and $\eta(\chi)$ is the conformal time corresponds to the comoving distance of $\chi$. Actually, the lensing potential is not a direct observable in CMB observations, but we can reconstruct it with observed lensed $\mathrm{CMB}$ anisotropies. It contains much more information than the lensed power spectrum [16,17], since the reconstruction is performed by making use of off-diagonal components in correlation function of lensed anisotropies [28]. In Fig. 3, we show the angular power spectra of the lensing potential and its correlation with the temperature anisotropy, $C_{\ell}^{\phi \phi}$ and $C_{\ell}^{T \phi}$, respectively. For reference, we also show expected data of future CMB experiments: Planck, PolarBeaR and CMBpol. We can see from the $C_{\ell}^{\phi \phi}$ in the Fig. 3 that the suppression of the power spectra depends on the mass of gravitino, which can be probed with future observations of CMB. When we carefully observe the power on small scales, the trend how the power is suppressed is similar to what 
we have seen in the matter power spectra. Therefore we can expect the mass of gravitino is constrained with reconstructed lensing potential, which would be obtained from future CMB surveys. Although cross correlation of the lensing potential with CMB temperature anisotropy $C_{\ell}^{T \phi}$ is affected by the mass of gravitino, the effect is much small compared with the expected errors for future CMB surveys, which can be seen from the right panel in Fig. 3. Thus it is suggested that $C_{\ell}^{T \phi}$ has little advantage for constraining the mass of light gravitino.

Here it should be noted that some careful consideration must be given for the following fact: the heavier the light gravitino mass is, the smaller the free-streaming scale would be. Although, regarding the suppression of the power, the effects of the light gravitino is more significant for a larger mass, when a survey cannot observe up to high multipoles due to its limitation of the resolution, gravitino with lighter mass can be better probed. This is because gravitino with lighter mass can erase cosmic structure up to larger scales compared to the case with larger mass although the power suppression is milder. As a simple example, let us compare the two cases, $f_{3 / 2}=0$ and $f_{3 / 2}=1$ while keeping $\omega_{\mathrm{dm}}$ fixed. At small angular scales, the lensing potential $\phi$ for $f_{3 / 2}=1$ is more suppressed than that for $f_{3 / 2}=0$. However, for the case with $f_{3 / 2}=1$ corresponding to $m_{3 / 2} \simeq 86 \mathrm{eV}$, the free streaming length is small. Therefore the suppression occurs only at limited small angular scales. If the observed multipoles are limited to low $\ell$ 's, where the suppression cannot be seen, gravitino with $f_{3 / 2}=1$ cannot leave any imprint on such a measurement, which means that we cannot differentiate models between $f_{3 / 2}=1$ and $f_{3 / 2}=0$. This makes the likelihood surface multi-modal and highly-degenerate. To break up these degeneracies, high-resolution measurement of lensing potential is needed, and currently available observations cannot suffice this requirement. In the next section, we discuss how future CMB surveys will constrain the light gravitino models.

\section{Constraints on light gravitino mass}

Now in this section, we investigate the constraints on the light gravitino mass from future CMB surveys. As discussed in the previous section, since the current CMB surveys are not precise enough to measure the lensing potential, it is almost impossible to probe $m_{3 / 2}$. However, in future surveys of $\mathrm{CMB}$, the measurement of lensing potential would be significantly improved. To forecast constraints on light gravitino mass from future CMB surveys, we make use of the following three surveys in this paper, the Planck [18], PolarBeaR [19] and CMBpol [20]. The parameters for instrumental design for these surveys are summarized in Table 1, where $\theta_{F W H M}$ is the size of Gaussian beam\#3 at FWHM and $\sigma_{T}\left(\sigma_{P}\right)$ is the temperature (polarization) noise.

In this paper, to generate samples from the Bayesian posterior distributions of cosmological parameters, we make use of the public code MultiNest [21] integrated in the vastly

${ }^{\# 3}$ We assume Gaussian beam and neglect any anisotropies in beam and distortion arising from the scan strategy. 


\begin{tabular}{l||c|c|c|c|c}
\hline \hline surveys & $f_{\text {sky }}$ & bands [GHz] & $\theta_{\text {FwhM }}[\operatorname{arcmin}]$ & $\sigma_{T}[\mu \mathrm{K}]$ & $\sigma_{P}[\mu \mathrm{K}]$ \\
\hline Planck [18] & 0.65 & 100 & 9.5 & 6.8 & 10.9 \\
& & 143 & 7.1 & 6.0 & 11.4 \\
& & 217 & 5.0 & 13.1 & 26.7 \\
\hline PolarBeaR [19] & 0.03 & 90 & 6.7 & 1.13 & 1.6 \\
& & 150 & 4.0 & 1.70 & 2.4 \\
& & 220 & 2.7 & 8.00 & 11.3 \\
\hline CMBpol [20] & \multirow{2}{*}{0.65} & 100 & 4.2 & 0.84 & 1.18 \\
& & 150 & 2.8 & 1.26 & 1.76 \\
& & 220 & 1.9 & 1.84 & 2.60 \\
\hline \hline
\end{tabular}

Table 1: Instrumental parameters for future CMB surveys used in our analysis. $\theta_{\text {FWHM }}$ is Gaussian beam width at FWHM, $\sigma_{T}$ and $\sigma_{P}$ are temperature and polarization noise, respectively. For the Planck and PolarBeaR surveys, we assume 1-year duration of observation and for the CMBpol survey, we assumed 4-year duration.

used Monte Carlo sampling code COSMOMC [22]. While COSMOMC samples the posterior distributions via the Markov chain Monte Carlo (MCMC) sampling method, MultiNest is based on the different sampling called nested-sampling method [23]. Use of MultiNest has several advantages in our analysis. One of the greatest advantages is that it enables efficient exploring of multi-modal/highly-degenerate likelihood surface, which is indeed the case for light gravitino models, as we have discussed in the previous section. Also it provides Bayesian evidence of a model and hence enables us to employ Bayesian model selection.

To obtain the limit for the mass of light gravitino, we can translate the constraint on the parameter $f_{3 / 2}=\omega_{3 / 2} / \omega_{\mathrm{dm}}$ using Eq. (5). Since light gravitino has almost definite prediction of its abundance, we mainly report our results for the case with $N_{3 / 2}=0.059$ being fixed. However, in some scenario such as those with late-time entropy production, this number may be altered. In this respect, we also make analysis with $N_{3 / 2}$ being varied. Furthermore, regarding the treatment of the primordial abundance of ${ }^{4} \mathrm{He}$ (denoted as $Y_{p}$ ), we assume two cases: treating $Y_{p}$ as a free parameter and fixing $Y_{p}$ with the derived value via the big bang nucleosynthesis (BBN) relation [24]. In the BBN theory, $Y_{p}$ is determined once baryon density $\omega_{\mathrm{b}}$ and the effective number of neutrino $N_{\text {eff }}$ are given. Thus such a fixing of the value of $Y_{p}$ was adopted in some analysis [25-27]. Since, in the precise measurement of future CMB survey, the prior on $Y_{p}$ can also affect the determination of other cosmological parameters [26,27], we consider the case with $Y_{p}$ freely varied as well.

Thus the full parameter space that we explore for light gravitino models are basically nine-dimensional:

$$
\left(\omega_{b}, \omega_{\mathrm{dm}}, \theta_{s}, \tau, N_{3 / 2}, f_{3 / 2}, Y_{p}, n_{s}, A_{s}\right),
$$

where $\theta_{s}$ is the acoustic peak scale, $\tau$ is the optical depth of reionization and $A_{s}$ and $n_{s}$ 


\begin{tabular}{l||c|c|c}
\hline \hline \multirow{2}{*}{ parameters } & & \multicolumn{2}{|c}{ prior ranges } \\
\cline { 3 - 4 } & fiducial values & Planck/PolarBeaR/combined & CMBpol \\
\hline$\omega_{b}$ & 0.02273 & $0.018 \rightarrow 0.28$ & $0.021 \rightarrow 0.024$ \\
$\omega_{\mathrm{dm}}$ & 0.1099 & $0.08 \rightarrow 0.30$ & $0.10 \rightarrow 0.14$ \\
$\theta_{s}$ & 1.0377 & $1.02 \rightarrow 1.06$ & $1.03 \rightarrow 1.04$ \\
$\tau$ & 0.087 & $0.01 \rightarrow 0.30$ & $0.06 \rightarrow 0.14$ \\
$N_{3 / 2}$ & 0.059 & $(0 \rightarrow 5)$ & $(0 \rightarrow 2)$ \\
$f_{3 / 2}$ & 0.013 & $0 \rightarrow 1$ & $0 \rightarrow 0.1$ \\
$Y_{p}$ & 0.248 & $(0.1 \rightarrow 0.5)$ & $(0.2 \rightarrow 0.3)$ \\
$n_{s}$ & 0.963 & $0.8 \rightarrow 1.2$ & $0.9 \rightarrow 1$ \\
$\ln \left(10^{10} A_{s}\right)$ & 3.063 & $2.8 \rightarrow 3.5$ & $3.0 \rightarrow 3.2$ \\
\hline \hline
\end{tabular}

Table 2: The fiducial values and prior ranges for the parameters used in the analysis. Note that priors shown with parenthesis are imposed only when the corresponding parameters $\left(N_{3 / 2}\right.$ and $\left.Y_{p}\right)$ are treated as free parameters and not imposed when they are fixed or derived from other parameters. For CMBpol, we take narrower range for the top priors since its accuracy is much higher than the former two surveys. Hence we do not need broad range for the priors.

are the amplitude and spectral index of initial power spectrum of scalar perturbations at a pivot scale $k_{0}=0.05 \mathrm{Mpc}^{-1}$. In the following, we investigate four different cases: (I) fixing $N_{3 / 2}=0.059$ and deriving $Y_{p}$ via the BBN relation, (II) fixing $N_{3 / 2}=0.059$ and treating $Y_{p}$ as a free parameter, (III) treating $N_{3 / 2}$ as a free parameter and $Y_{p}$ as a derived parameter via the BBN relation, and (IV) treating $N_{3 / 2}$ and $Y_{p}$ as free parameters. The fiducial values and top-hat priors for parameter estimation are summarized in Table 2 .

The likelihood function is adopted from Ref. [9]. We include TT, TE, EE, $\phi \phi, T \phi$ spectra for correlation of CMB anisotropy and lensing potential up to $\ell \leq 2500$. We assume lensing reconstruction is performed by adopting the method based on quadratic estimator [28], and the expected noise in lensing potential is calculated by the publicly available FuturCMB2 code developed by the authors of [9]. Angular power spectra are calculated using the method in Ref. [29]. For corrections for lensing potential due to nonlinear evolution of matter density perturbations, we adopt HALOFIT, which is based on the N-body simulations of CDM models [30]. Though the light gravitino model is not exactly the CDM models, we believe that the change of the nonlinear correction is negligible. This is because that gravitino has small $N_{3 / 2}$ and regardless of the mass of $m_{3 / 2}$, dark matter can be approximated by CDM very well when it begins to evolve in nonlinear regime. Furthermore, nonlinear correction changes the spectra of lensing potential by only a few percent at $\ell \leq 2500$ [29], and hence the treatment here can be justified. In addition, we also performed same analyses without including nonlinear corrections and checked that resultant constraints do not significantly change by the treatment of nonlinearity.

Now we are going to present our results. In Tables 3 , 6 we summarize the constraints on 
the cosmological parameters from Planck alone, PolarBeaR alone, Planck and PolarBeaR combined, and CMBpol alone, separately for different priors. First we discuss the case with fixed $N_{3 / 2}=0.059$ and the BBN relation adopted for $Y_{p}$. The $1 \mathrm{~d}$ posterior distributions of cosmological parameters are shown in Fig. 4. From the posterior distributions for $f_{3 / 2}$ in Fig. 目, we can easily see that light gravitino models are not constrained very well with Planck or PolarBeaR alone. The posterior distributions have decaying tails from the peak near $f_{3 / 2}=0$ to $f_{3 / 2}=1$. Actually, they have very smooth second peaks at around $f_{3 / 2}=1$. This multi-modal structure of posterior distributions comes from the degeneracies what we have discussed in Section 3, Light gravitino with a relatively large mass $\left(f_{3 / 2} \simeq 1\right)$ can suppress the power via free-streaming only at very small scales where the Planck surveys cannot sufficiently measure the CMB. Thus a model with such a gravitino mass can fit the data from Planck alone. On the other hand, PolarBeaR has better resolution, gravitino with large mass is much constrained from observation of lensing potential at small scales. However, the sky coverage of the PolarBeaR survey is much smaller than Planck, thus the observation is worse at large angular scales. In this case, other parameters than $f_{3 / 2}$ are still not well-constrained from PolarBeaR alone. Therefore gravitino with relatively large mass can fit the data to some extent by tuning other cosmological parameters in this case too. To remove the degeneracy it is necessary to combine observations precise at large and small angular scales or, ultimately, use measurements precise both at large and small angular scales. With data from Planck and PolarBeaR combined, we can obtain a constraint

$$
f_{3 / 2} \leq 0.036 \text { (95\% C.L.). }
$$

When we use CMBpol, whose measurement is precise both on large and small scales than other two survey, this constraint can be improved as

$$
f_{3 / 2}=0.0121 \pm 0.0027 \text { (68\% C.L.) }
$$

These constraints are translated into the limits on the mass of light gravitino. For the case with Planck and PolarBeaR combined, the constraint is given as

$$
m_{3 / 2} \leq 3.2 \mathrm{eV} \text { (95\% C.L.) }
$$

and with CMBpol as

$$
m_{3 / 2}=1.04_{-0.26}^{+0.22} \mathrm{eV} \text { (68\% C.L.). }
$$

Since gravitino mass should be larger than $1 \mathrm{eV}$ not to destabilize the messenger scalar, CMBpol would be expected to give (counter-)evidense for existence of gravitino if its mass is (not) in the mass range considered here. In Section 5 we will discuss this point more quantitatively using Bayesian models selection analysis.

So far we kept assuming the energy density of gravitino fixed as $N_{3 / 2}=0.059$. If we loosen this assumption and take $N_{3 / 2}$ as a free parameter, the constraints are significantly weakened. Notice that the free-streaming scale of light gravitino is determined by its mass. Thus when $N_{3 / 2}$ is freely varied and hence the mass of gravitino can be large, the 
free-streaming scale can be shifted toward smaller scales over which PolarBeaR cannot observe for a wide range of the mass and one cannot see the damping of the power there. (On the other hand, since CMBpol is very precise on small scales, the constraint on $m_{3 / 2}$ from CMBpol is not affected much.) Thus we cannot obtain a meaningful constraint on $f_{3 / 2}$ even if we combine Planck and PolarBeaR when we take $N_{3 / 2}$ as a free parameter. Furthermore, the change in $N_{3 / 2}$ renders the shift of the radiation-matter equality which can be absorbed by tuning $\omega_{\mathrm{dm}}$ [31-33], significant degeneracies arise among $\omega_{\mathrm{dm}}, f_{3 / 2}$ and $N_{3 / 2}$ as shown in Fig. [0 $1 \# 4$. For meaningful constraints we need sensitivities as good as those of CMBpol-like survey. In Fig. 6 1d posterior distributions for parameters including $N_{3 / 2}$ are shown. The $68 \%$ limit of $f_{3 / 2}$ for this case is $f_{3 / 2}=0.0118_{-0.0031}^{+0.0032}$, which corresponds to the constraint on the gravitino mass $m_{3 / 2}=1.19_{-0.50}^{+0.16} \mathrm{eV}$.

\begin{tabular}{l||cccc}
\hline \hline parameters & Planck & PolarBeaR & combined & CMBpol \\
\hline $100 \omega_{b}$ & $2.276_{-0.012}^{+0.013}$ & $2.274_{-0.022}^{+0.021}$ & $2.275_{-0.009}^{+0.012}$ & $2.2739_{-0.0033}^{+0.00041}$ \\
$\omega_{d m}$ & $0.1101_{-0.0013}^{+0.0010}$ & $0.1106_{-0.0022}^{+0.0022}$ & $0.1100_{-0.0012}^{+0.00010}$ & $0.10993_{-0.00005}^{+0.00059}$ \\
$100 \theta_{s}$ & $103.783_{-0.026}^{+0.024}$ & $103.777_{-0.035}^{+0.035}$ & $103.777_{-0.019}^{+0.020}$ & $103.774_{-0.0056}^{+0.0056}$ \\
$\tau$ & $0.0871_{-0.0045}^{+0.0043}$ & $0.090_{-0.013}^{+0.009}$ & $0.0873_{-0.0043}^{+0.0039}$ & $0.0872_{-0.0026}^{+0.0022}$ \\
$N_{3 / 2}$ & - & - & - & - \\
$f_{3 / 2}$ & - & - & $<0.036(95 \%)$ & $0.0121_{-0.0027}^{+0.0027}$ \\
$Y_{p}$ & - & - & - & - \\
$n_{s}$ & $0.9622_{-0.0036}^{+0.0045}$ & $0.9621_{-0.0083}^{+0.0081}$ & $0.9629_{-0.0033}^{+0.0036}$ & $0.9637_{-0.0017}^{+0.0017}$ \\
$\ln \left(10^{10} \times A_{s}\right)$ & $3.0640_{-0.0094}^{+0.0073}$ & $3.077_{-0.026}^{+0.015}$ & $3.0641_{-0.0094}^{+0.0063}$ & $3.0637_{-0.0047}^{+0.00040}$ \\
\hline$m_{3 / 2}[\mathrm{eV}]$ & - & - & $<3.2(95 \%)$ & $1.04_{-0.26}^{+0.22}$ \\
\hline \hline
\end{tabular}

Table 3: Constraints on cosmological parameters for the case with fixing $N_{3 / 2}=0.059$ and adopting the BBN relation to fix the value of $Y_{p}$ (CASE I). We basically present the mean values as well as $1 \sigma$ errors. For parameters that are bounded only from one side we present $95 \%$ credible intervals.

\section{$5 \quad$ Model selection analysis on light gravitino model}

In the previous section, we have seen that future $\mathrm{CMB}$ surveys give rather tight constraints on mass of light gravitino, so that we can expect they would give (counter-)evidence for existence of gravitino to more or less extent. But then a question arises how strong the evidence for gravitino is. This is a kind of model selection problem in statistics theory, which has been often argued in cosmology [34-45]. In Bayesian statistics, the natural

\footnotetext{
\#4 In Figure 7 and 12 in [3], a similar degeneracy can also be seen as band-like allowed region along $F_{\text {WDM }}$ axis.
} 


\begin{tabular}{|c|c|c|c|c|}
\hline parameters & Planck & PolarBeaR & combined & CMBpol \\
\hline $100 \omega_{b}$ & $2.278_{-0.017}^{+0.021}$ & $2.272_{-0.034}^{+0.035}$ & $2.274_{-0.014}^{+0.016}$ & $2.2722_{-0.0047}^{+0.0055}$ \\
\hline$\omega_{d m}$ & $0.1100_{-0.0010}^{+0.0015}$ & $0.1107_{-0.0021}^{+0.0025}$ & $0.1100_{-0.0011}^{+0.0011}$ & $0.10990_{-0.00056}^{+0.00061}$ \\
\hline $100 \theta_{s}$ & $103.788_{-0.042}^{+0.048}$ & $103.775_{-0.058}^{+0.067}$ & $103.775_{-0.035}^{+0.032}$ & $103.771_{-0.011}^{+0.011}$ \\
\hline$\tau$ & $0.0873_{-0.0050}^{+0.0041}$ & $0.090_{-0.013}^{+0.011}$ & $0.0873_{-0.0044}^{+0.0040}$ & $0.0870_{-0.0028}^{+0.0020}$ \\
\hline$N_{3 / 2}$ & - & - & - & - \\
\hline$f_{3 / 2}$ & - & - & $<0.035(95 \%)$ & $0.0118_{-0.0025}^{+0.0030}$ \\
\hline$Y_{p}$ & $0.250_{-0.011}^{+0.008}$ & $0.248_{-0.016}^{+0.016}$ & $0.2486_{-0.0066}^{+0.0094}$ & $0.2481_{-0.0028}^{+0.0031}$ \\
\hline$n_{s}$ & $0.9627_{-0.0063}^{+0.0067}$ & $0.961_{-0.014}^{+0.012}$ & $0.9625_{-0.0052}^{+0.0063}$ & $0.9629_{-0.0025}^{+0.0026}$ \\
\hline $\ln \left(10^{10} \times A_{s}\right)$ & $3.064_{-0.010}^{+0.008}$ & $3.071_{-0.028}^{+0.018}$ & $3.064_{-0.010}^{+0.006}$ & $3.0631_{-0.0045}^{+0.0043}$ \\
\hline$m_{3 / 2}[\mathrm{eV}]$ & - & - & $<3.1(95 \%)$ & $1.02_{-0.22}^{+0.26}$ \\
\hline
\end{tabular}

Table 4: Same tables as in Table 3 but for the cases with fixing $N_{3 / 2}=0.059$ and treating $Y_{p}$ as a free parameter (CASE II).

measure for evidence of a model is Bayesian evidence $E$,

$$
E(M)=\int d \theta P(\operatorname{data} \mid \theta) \pi(\theta \mid M)
$$

where $\theta$ represents a set of parameters included in a model $M . P($ data $\mid \theta)$ and $\pi(\theta \mid M)$ are the likelihood and prior probability functions, respectively. Bayesian evidence can be efficiently calculated by nested sampling method [23]. Predictiveness of a model $M_{1}$ against another $M_{2}$ can be assessed by differencing the logarithm of Bayes factors of the models, that is

$$
B_{12}=\ln \left(E\left(M_{1}\right) / E\left(M_{2}\right)\right)
$$

which is called Bayes factor. If $B_{12}$ is positively (negatively) large, we can say the observed data can be explained well by model $M_{1}\left(M_{2}\right)$ compared with $M_{2}\left(M_{1}\right)$. As a rule of thumb the Jeffreys' scale is often used to translate a Bayes factor into literal expression for strength of an evidence: $B_{12}<1$ is not significant, $1<B_{12}<2.5$ significant, $2.5<B_{12}<5$ strong and $5<B_{12}$ is decisive. For more details we refer to a recent review [45] and references therein.

Now we are going to see how large the evidence is from future CMB surveys. In Table 7 we summarized values of obtained Bayes factor for light gravitino model with different sets of data and priors against the conventional CDM model $\left(f_{3 / 2}=N_{3 / 2}=0\right)$. Here we have assumed a same fiducial model $\left(m_{3 / 2}=1 \mathrm{eV}\right.$ and $\left.N_{3 / 2}=0.059\right)$, as in the previous section.

First of all, from Table 7 we can see that Planck or PolarBeaR alone and even Planck and PolarBeaR combined give only negative Bayes factor for the gravitino model against the CDM model, regardless of priors on $Y_{p}$ and $N_{3 / 2}$. This is because for most of values of added parameters $f_{3 / 2}$ (and $N_{3 / 2}$ ) from the CDM model, gravitino model can only marginally improve fit to the data, even though it indeed improves the fit at some values 


\begin{tabular}{l||cccc}
\hline \hline parameters & Planck & PolarBeaR & combined & CMBpol \\
\hline $100 \omega_{b}$ & $2.276_{-0.015}^{+0.015}$ & $2.284_{-0.030}^{+0.023}$ & $2.276_{-0.014}^{+0.011}$ & $2.2735_{-0.0048}^{+0.0043}$ \\
$\omega_{d m}$ & $0.1099_{-0.00034}^{+0.00034}$ & $0.1125_{-0.00060}^{+0.0036}$ & $0.1097_{-0.00033}^{+0.0017}$ & $0.1097_{-0.0011}^{+0.0011}$ \\
$100 \theta_{s}$ & $103.782_{-0.029}^{+0.035}$ & $103.763_{-0.038}^{+0.053}$ & $103.778_{-0.027}^{+0.027}$ & $103.776_{-0.00982}^{+0.0092}$ \\
$\tau$ & $0.0873_{-0.0047}^{+0.0040}$ & $0.091_{-0.015}^{+0.008}$ & $0.0874_{-0.00046}^{+0.0036}$ & $0.0871_{-0.0027}^{+0.0022}$ \\
$N_{3 / 2}$ & $<0.24(95 \%)$ & $<0.47(95 \%)$ & $<0.21(95 \%)$ & $<0.10(95 \%)$ \\
$f_{3 / 2}$ & - & - & - & $0.0118_{-0.0031}^{+0.0032}$ \\
$Y_{p}$ & - & - & - & - \\
$n_{s}$ & $0.9638_{-0.0065}^{+0.0034}$ & $0.968_{-0.012}^{+0.009}$ & $0.9639_{-0.0054}^{+0.0038}$ & $0.9635_{-0.0025}^{+0.0020}$ \\
$\ln \left(10^{10} \times A_{s}\right)$ & $3.064_{-0.010}^{+0.009}$ & $3.076_{-0.027}^{+0.021}$ & $3.064_{-0.010}^{+0.008}$ & $3.0632_{-0.0049}^{+0.0051}$ \\
\hline$m_{3 / 2}[\mathrm{eV}]$ & - & - & - & $1.19_{-0.50}^{+0.16}$ \\
\hline \hline
\end{tabular}

Table 5: Same tables as in Table 3 but for the cases with treating $N_{3 / 2}$ as a free parameter and adopting the BBN relation (CASE III).

around the fiducial ones, $f_{3 / 2} \simeq 0.013$ (and $N_{3 / 2} \simeq 0.059$ ). In other words, the complexity of the gravitino model has little advantage in explaining the data.

The situation dramatically changes for the case of the CMBpol survey. From Table 7 we can see from CMBpol data we obtain Bayes factor for gravitino model against the CDM model as $\ln B=3.40 \pm 0.16$ for a case with fixing $N_{3 / 2}=0.059$ and using the $\mathrm{BBN}$ relation. This is interpreted as strong evidence in the Jeffreys' scale, though there's always some disagreement in that how much Bayes factor can be regarded as giving enough evidence. For the cases with treating $N_{3 / 2}$ as a free parameter and using the BBN relation, we obtain $\ln B \gtrsim 1.08 \pm 0.17$. This can be regarded as giving only marginal evidence. So it is difficult to obtain enough evidence for general WDM model whose number density is not theoretically limited in some small range. Fortunately, since gravitino has small model-dependence of $N_{3 / 2}$ we can take the former value of $\ln B$.

So far we have discussed the case of fiducial gravitino mass $m_{3 / 2}$. For larger gravitino mass, as long as it is less than the current bound $\left(m_{3 / 2} \lesssim 16 \mathrm{eV}[2,3]\right)$, the evidence surely improves. This is because for this range of mass, the power spectra of lensing potential differ more and more from those for the CDM model as the gravitino mass increases. Since, in Section 2 we have seen that gravitino mass is expected to be $O(1)$ eV or larger theoretically, the fiducial model of $m_{3 / 2}=1 \mathrm{eV}$, which we used throughout this paper, can be supposed as a rather pessimistic case. Since we have seen that even evidence for gravitino with mass $1 \mathrm{eV}$ can be probed by a CMBpol-like survey, we would expect such a survey can probe most part of theoretically-motivated range of light gravitino mass. We hope such a survey would be realized and probe light gravitino model in the near future. 


\begin{tabular}{l||cccc}
\hline \hline parameters & Planck & PolarBeaR & combined & CMBpol \\
\hline $100 \omega_{b}$ & $2.278_{-0.021}^{+0.017}$ & $2.276_{-0.035}^{+0.030}$ & $2.273_{-0.013}^{+0.012}$ & $2.2727_{-0.0056}^{+0.0046}$ \\
$\omega_{d m}$ & $0.1117_{-0.0023}^{+0.005}$ & $0.1176_{-0.00692}^{+0.0062}$ & $0.1103_{-0.0014}^{+0.00011}$ & $0.1103_{-0.0016}^{+0.0013}$ \\
$100 \theta_{s}$ & $103.75_{-0.06}^{+0.10}$ & $103.65_{-0.14}^{+0.14}$ & $103.760_{-0.045}^{+0.068}$ & $103.764_{-0.019}^{+0.027}$ \\
$\tau$ & $0.0875_{-0.0049}^{+0.0042}$ & $0.091_{-0.015}^{+0.008}$ & $0.0872_{-0.0031}^{+0.0036}$ & $0.0872_{-0.0025}^{+0.0021}$ \\
$N_{3 / 2}$ & $<0.47(95 \%)$ & $<1.3(95 \%)$ & $<0.34(95 \%)$ & $<0.17(95 \%)$ \\
$f_{3 / 2}$ & - & - & - & $0.0124_{-0.0034}^{+0.0032}$ \\
$Y_{p}$ & $0.2458_{-0.011}^{+0.015}$ & $0.230_{-0.018}^{+0.029}$ & $0.246_{-0.007}^{+0.0010}$ & $0.2471_{-0.0043}^{+0.0043}$ \\
$n_{s}$ & $0.9643_{-0.0068}^{+0.0068}$ & $0.965_{-0.013}^{+0.014}$ & $0.9636_{-0.0045}^{+0.0047}$ & $0.9632_{-0.0027}^{+0.0024}$ \\
$\ln \left(10^{10} \times A_{s}\right)$ & $3.067_{-0.011}^{+0.009}$ & $3.082_{-0.032}^{+0.019}$ & $3.0636_{-0.0077}^{+0.0079}$ & $3.0641_{-0.0053}^{+0.0046}$ \\
\hline$m_{3 / 2}[\mathrm{eV}]$ & - & - & - & $1.10_{-0.61}^{+0.07}$ \\
\hline \hline
\end{tabular}

Table 6: Same tables as in Table 3 but for the cases with treating both $N_{3 / 2}$ and $Y_{p}$ as free parameters (CASE IV).

\begin{tabular}{lrrrr}
\hline \hline datasets & CASE I & CASE II & CASE III & CASE IV \\
\hline Planck alone & $-3.36 \pm 0.17$ & $-2.81 \pm 0.17$ & $-5.76 \pm 0.17$ & $-4.70 \pm 0.18$ \\
PolarBeaR alone & $-3.39 \pm 0.15$ & $-2.66 \pm 0.18$ & $-5.02 \pm 0.16$ & $-3.62 \pm 0.18$ \\
combined & $-3.03 \pm 0.17$ & $-2.86 \pm 0.18$ & $-5.80 \pm 0.17$ & $-5.82 \pm 0.18$ \\
CMBpol alone & $3.40 \pm 0.16$ & $3.63 \pm 0.17$ & $1.08 \pm 0.17$ & $1.44 \pm 0.19$ \\
\hline \hline
\end{tabular}

Table 7: Bayes factors for the light gravitino model against the CDM model. Shown are the mean and standard errors from two independent samplings.

\section{Summary and discussion}

We investigated a possible constraint on the light gravitino mass with $m_{3 / 2}<100 \mathrm{eV}$ in the light of future precise measurements of CMB. Although the effects of free-streaming of light gravitino barely leave an imprint on CMB photons at the time of last scatter, they can be deflected by the gravitational potential altered after recombination due to the freestreaming effect, which can be probed with lensed CMB. Thus we in this paper discussed the effects of the light gravitino, paying particular attention to the lensing potential, then investigated the future constraint on its mass. For this purpose, we adopt the future CMB surveys such as Planck, PolarBeaR and CMBpol and study the issue by generating posterior distributions with nested-sampling method. For a simple (but physically motivated) case, we obtained the limit on the light gravitino mass, assuming $m_{3 / 2}=1 \mathrm{eV}$ as a fiducial value, as $m_{3 / 2} \leq 3.2 \mathrm{eV}$ (95\% C.L.) for the case with Planck+PolarBeaR combined and $m_{3 / 2}=1.04_{-0.26}^{+0.22} \mathrm{eV}(68 \%$ C.L. $)$ for CMBpol. Thus at the time of CMBpol experiment, we can expect that the (counter-)evidence of the light gravitino can be found in cosmological observations. 

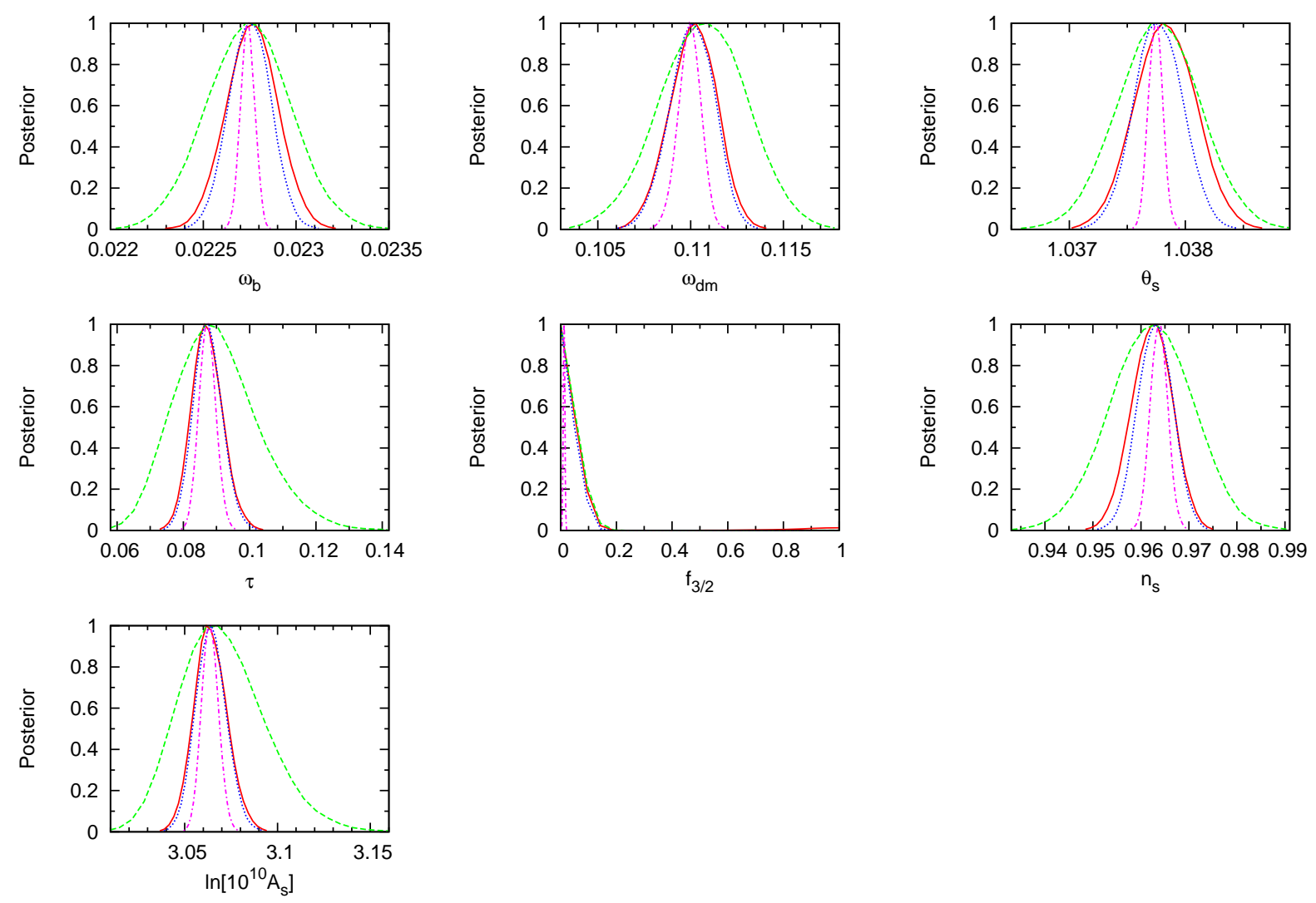

Figure 4: The 1d posterior distributions for cosmological parameters with fixed $N_{3 / 2}=$ 0.059 and the BBN relation for $Y_{p}$ adopted. Shown are constraints from Planck alone (red solid line), PolarBeaR alone (green dashed line), Planck and PolarBeaR combined (blue dotted line), and CMBpol (magenta dash-dotted line).

In a simple case, the effective degrees of freedom at the time of the gravitino decoupling is assumed to have definite value and fixed in the analysis. However in some scenarios, this assumption may not hold, thus we have also made analysis by treating $N_{3 / 2}$ as a free parameter. In addition, in the future precise CMB measurements, the primordial abundance of ${ }^{4} \mathrm{He}$, which is usually not assumed as a free parameter but fixed, can also affect the determination of cosmological parameters, therefore we also performed the analysis by varying $Y_{p}$ too. When both or one of these parameters are varied, the constraint on the mass becomes weak. The results are summarized in Tables [3, 6. In addition, we also discussed how strong future CMB surveys can find an evidence for light gravitino by employing Bayesian model selection analysis. Even if the mass of gravitino is around $1 \mathrm{eV}$, a future CMBpol-like surveys is capable of providing some rather strong evidence for light gravitino model, which can be even stronger for larger $m_{3 / 2}$.

In principle the light gravitino mass of $\mathcal{O}(1) \mathrm{eV}$ can be probed with the LHC experiment 

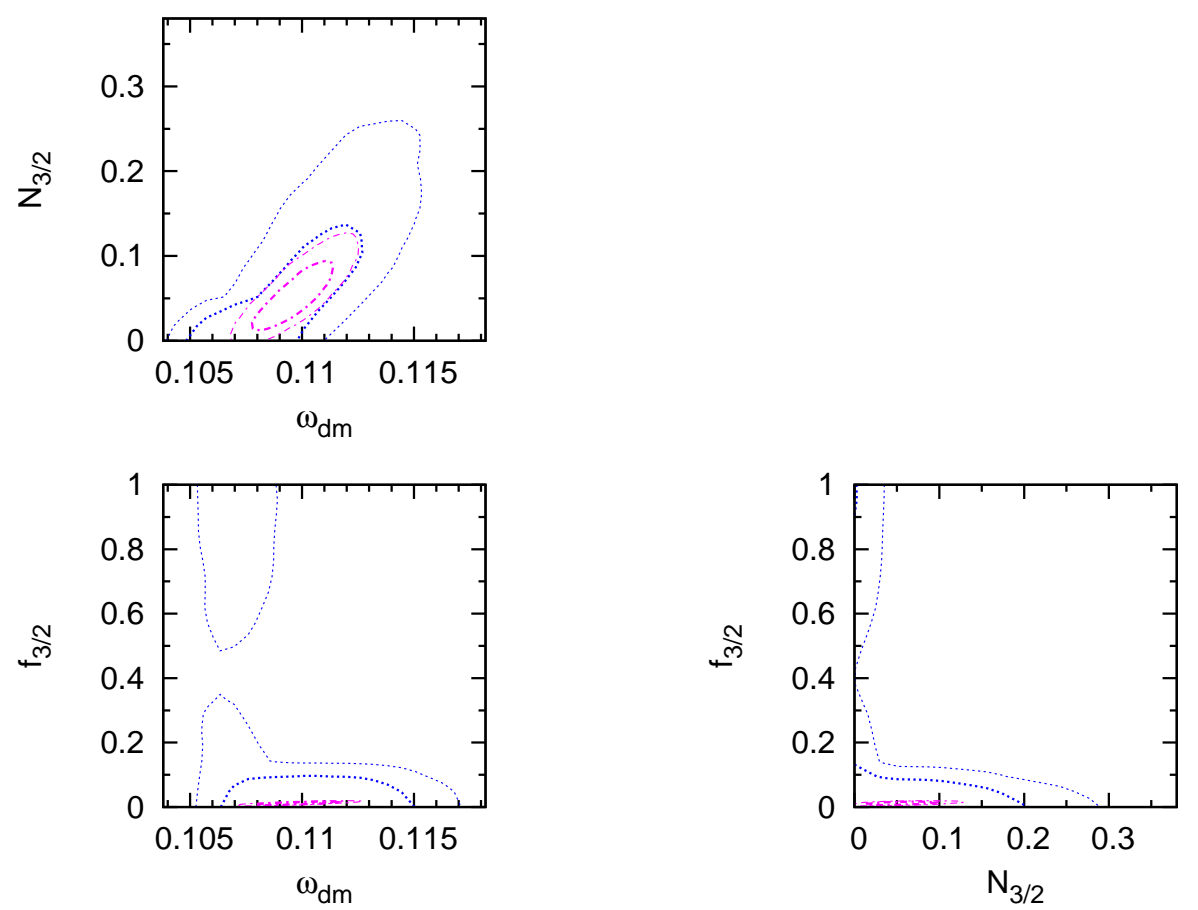

Figure 5: The degeneracies arise among $\omega_{\mathrm{dm}}, f_{3 / 2}$ and $N_{3 / 2}$. Shown are the cases where we adopted the BBN relation for $Y_{p}$ using data from Planck and PolarBeaR combined (blue dotted line) and CMBpol alone (magenta dash-dotted line). The thick and thin lines show contours at $68 \%$ and $95 \%$ C.L., respectively

with the method proposed in Ref. [1]. But this requires some amount of tuning for the sparticle mass spectrum together with the gravitino mass. Thus even if the LHC will fail to determine the light gravitino mass, the future CMB experiments can do this job.

Finally we make some comment on the case where massive neutrinos are also included in the analysis. From atmospheric, solar, reactor and accelerator neutrino experiments, now we know that neutrinos have finite masses. Furthermore it has been discussed that neutrino masses can be well probed with future CMB survey [7-9], which motivates us to conduct the analysis assuming that neutrinos are massive. Thus we also investigated the constraint on the light gravitino mass while the mass of neutrino is also varied. Since the effects of massive neutrino and light gravitino on the lensing potential are essentially the same, we found that strong degeneracies arise in particular, between their masses and we could not obtain any meaningful constraints on those. However, neutrino masses can also be constrained in future laboratory experiments such as tritium beta-decay and neutrinoless double beta decay. Thus in the future, we will also have some inputs from such neutrino experiments, which can remove the degeneracy in CMB survey. In light of these considerations, we can expect that cosmology and particle physics experiments will 

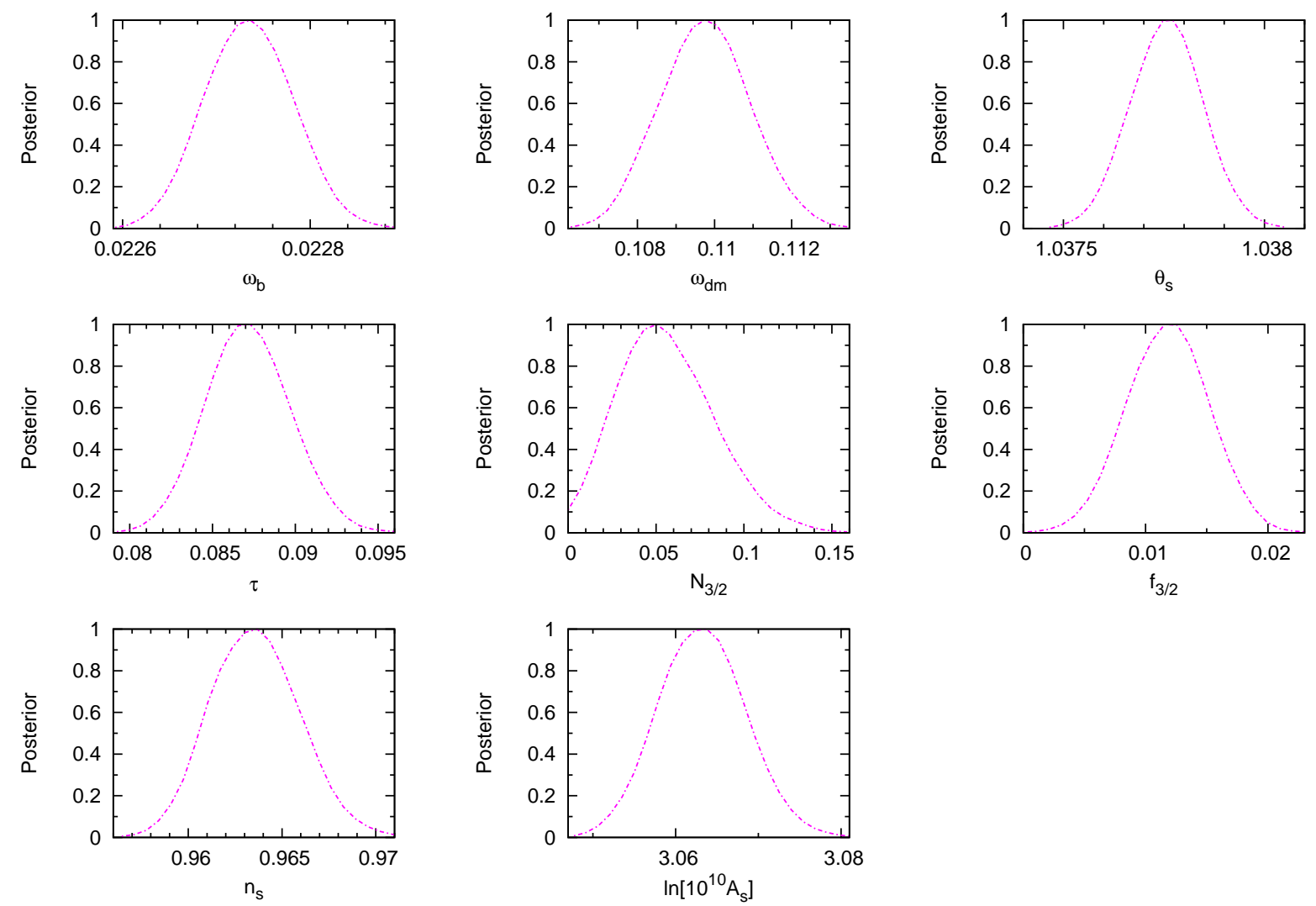

Figure 6: The 1d posterior distributions for cosmological parameters with freely varying $N_{3 / 2}$ and the BBN relation for $Y_{p}$ adopted. The case for CMBpol data is only shown.

push us toward more severe constraint/precise determination of the light gravitino mass in the near future.

\section{Acknowledgment}

The authors would like to thank Kiyotomo Ichiki and Shun Saito for useful discussions and providing data to check our numerical calculations. The authors would also like to thank Oleg Ruchayskiy for useful comments. This work is supported by Grant-in-Aid for Scientific research from the Ministry of Education, Science, Sports, and Culture, Japan, No. 14102004 (M.K.) and No. 19740145 (T.T.), and also by World Premier International Research Center Initiative, MEXT, Japan. K.N. and T.S. would like to thank the Japan Society for the Promotion of Science for financial support. 


\section{References}

[1] K. Hamaguchi, S. Shirai and T. T. Yanagida, Phys. Lett. B 651, 44 (2007) arXiv:0705.0219 [hep-ph]]; Phys. Lett. B 663, 86 (2008) [arXiv:0712.2462 [hep-ph]].

[2] M. Viel, J. Lesgourgues, M. G. Haehnelt, S. Matarrese and A. Riotto, Phys. Rev. D 71, 063534 (2005) arXiv:astro-ph/0501562.

[3] A. Boyarsky, J. Lesgourgues, O. Ruchayskiy and M. Viel, JCAP 0905, 012 (2009) arXiv:0812.0010 [astro-ph]].

[4] E. Komatsu et al. [WMAP Collaboration], Astrophys. J. Suppl. 180, 330 (2009) arXiv:0803.0547 [astro-ph]].

[5] M. Viel, M. G. Haehnelt and A. Lewis, Mon. Not. Roy. Astron. Soc. Lett. 370, L51 (2006) arXiv:astro-ph/0604310.

[6] U. Seljak, A. Slosar and P. McDonald, JCAP 0610, 014 (2006) arXiv:astro-ph/0604335.

[7] M. Kaplinghat, L. Knox and Y. S. Song, Phys. Rev. Lett. 91, 241301 (2003) arXiv:astro-ph/0303344.

[8] J. Lesgourgues, L. Perotto, S. Pastor and M. Piat, Phys. Rev. D 73, 045021 (2006) arXiv:astro-ph/0511735.

[9] L. Perotto, J. Lesgourgues, S. Hannestad, H. Tu and Y. Y. Y. Wong, JCAP 0610, 013 (2006) arXiv:astro-ph/0606227.

[10] For a review, see G. F. Giudice and R. Rattazzi, Phys. Rept. 322, 419 (1999) arXiv:hep-ph/9801271.

[11] K. Hamaguchi, S. Shirai and T. T. Yanagida, Phys. Lett. B 654, 110 (2007) arXiv:0707.2463 [hep-ph]].

[12] T. Moroi, H. Murayama and M. Yamaguchi, Phys. Lett. B 303, 289 (1993).

[13] M. Fukugita and T. Yanagida, Phys. Lett. B 174, 45 (1986); For a review, see W. Buchmuller, R. D. Peccei and T. Yanagida, Ann. Rev. Nucl. Part. Sci. 55, 311 (2005) arXiv:hep-ph/0502169.

[14] E. Pierpaoli, S. Borgani, A. Masiero and M. Yamaguchi, Phys. Rev. D 57, 2089 (1998) arXiv:astro-ph/9709047.

[15] A. Lewis and A. Challinor, Phys. Rept. 429, 1 (2006) arXiv:astro-ph/0601594. 
[16] K. M. Smith, W. Hu and M. Kaplinghat, Phys. Rev. D 74, 123002 (2006) arXiv:astro-ph/0607315.

[17] K. M. Smith et al., arXiv:0811.3916 [astro-ph].

[18] [Planck Collaboration], arXiv:astro-ph/0604069.

[19] http://bolo.berkeley.edu/polarbear

[20] D. Baumann et al. [CMBPol Study Team Collaboration], AIP Conf. Proc. 1141, 10 (2009) arXiv:0811.3919 [astro-ph]].

[21] F. Feroz and M. P. Hobson, arXiv:0704.3704 [astro-ph]. F. Feroz, M. P. Hobson and M. Bridges, arXiv:0809.3437 [astro-ph].

[22] A. Lewis and S. Bridle, Phys. Rev. D 66, 103511 (2002) arXiv:astro-ph/0205436.

[23] Skilling J., 2004, AIP Conference Proceedings of the 24th International Workshop on Bayesian Inference and Maximum Entropy Methods in Science and Engineering, Vol. 735, pp. 395-405

[24] L. H. Kawano, FERMILAB-Pub-92/04-A (1992).

[25] K. Ichikawa and T. Takahashi, Phys. Rev. D 73, 063528 (2006) [arXiv:astro-ph/0601099.

[26] J. Hamann, J. Lesgourgues and G. Mangano, JCAP 0803, 004 (2008) arXiv:0712.2826 [astro-ph]].

[27] K. Ichikawa, T. Sekiguchi and T. Takahashi, Phys. Rev. D 78, 043509 (2008) arXiv:0712.4327 [astro-ph]].

[28] T. Okamoto and W. Hu, Phys. Rev. D 67, 083002 (2003) arXiv:astro-ph/0301031.

[29] A. Challinor and A. Lewis, Phys. Rev. D 71, 103010 (2005) arXiv:astro-ph/0502425.

[30] R. E. Smith et al. [The Virgo Consortium Collaboration], Mon. Not. Roy. Astron. Soc. 341, 1311 (2003) arXiv:astro-ph/0207664.

[31] S. Hannestad and G. Raffelt, JCAP 0404, 008 (2004) arXiv:hep-ph/0312154.

[32] P. Crotty, J. Lesgourgues and S. Pastor, Phys. Rev. D 69, 123007 (2004) arXiv:hep-ph/0402049].

[33] K. Ichikawa, T. Sekiguchi and T. Takahashi, Phys. Rev. D 78, 083526 (2008) arXiv:0803.0889 [astro-ph]]. 
[34] A. Slosar et al., Mon. Not. Roy. Astron. Soc. 341, L29 (2003) arXiv:astro-ph/0212497.

[35] M. Beltran, J. Garcia-Bellido, J. Lesgourgues, A. R. Liddle and A. Slosar, Phys. Rev. D 71, 063532 (2005) arXiv:astro-ph/0501477.

[36] R. Trotta, Mon. Not. Roy. Astron. Soc. 378, 72 (2007) arXiv:astro-ph/0504022.

[37] P. Mukherjee, D. Parkinson and A. R. Liddle, Astrophys. J. 638, L51 (2006) arXiv:astro-ph/0508461.

[38] M. Bridges, A. N. Lasenby and M. P. Hobson, Mon. Not. Roy. Astron. Soc. 369, 1123 (2006) arXiv:astro-ph/0511573.

[39] M. Kunz, R. Trotta and D. Parkinson, Phys. Rev. D 74, 023503 (2006) arXiv:astro-ph/0602378.

[40] J. Magueijo and R. D. Sorkin, Mon. Not. Roy. Astron. Soc. Lett. 377, L39 (2007) arXiv:astro-ph/0604410.

[41] A. R. Liddle, P. Mukherjee and D. Parkinson, arXiv:astro-ph/0608184.

[42] C. Pahud, A. R. Liddle, P. Mukherjee and D. Parkinson, Mon. Not. Roy. Astron. Soc. 381, 489 (2007) arXiv:astro-ph/0701481.

[43] A. F. Heavens, T. D. Kitching and L. Verde, Mon. Not. Roy. Astron. Soc. 380, 1029 (2007) arXiv:astro-ph/0703191.

[44] N. Bevis, M. Hindmarsh, M. Kunz and J. Urrestilla, Phys. Rev. Lett. 100, 021301 (2008) arXiv:astro-ph/0702223.

[45] R. Trotta, Contemp. Phys. 49, 71 (2008) [arXiv:0803.4089 [astro-ph]]. 